\title{
逐层数据再表达的前后端融合学习的理论及其模型 和算法
}

\author{
郭田德 1,2 ，韩丛英 ${ }^{1,2^{*}}$ ，李明强 1,3 \\ 1. 中国科学院大学, 北京 100049 \\ 2. 中国科学院大数据挖掘与知识管理重点实验室, 北京 100190 \\ 3. 中国电子科技集团公司信息科学研究院, 北京 100086 \\ * 通信作者. E-mail: hancy@ucas.ac.cn
}

收稿日期: 2017-12-07；接受日期: 2018-01-28；网络出版日期: 2019-06-11

国家自然科学基金 (批准号: 11331012, 11731013, 11571014) 资助项目

摘要 基于学习的两个主要研究内容, 本文提出了学习的二元分层模式, 给出了前端学习、后端学 习、前后端组合学习和前后端融合学习的概念, 构建了前后端融合学习的理论框架与最优化模型; 针 对前端学习, 模拟大脑的分级工作机制, 提出了数据与模型混合驱动的逐层数据再表达的方法; 最 后, 以视觉 (图像) 学习为例, 本文给出了一种数据与模型混合驱动的逐层数据再表达的具体方法.

关键词 机器学习, 模式识别, 数据表达, 数据与模型混合驱动

\section{1 引言}

近几年, 作为人工智能的核心, 机器学习的应用已遍及人工智能的各个分支, 如专家系统、自动推 理、自然语言理解、模式识别、计算机视觉、智能机器人和自动驾驶等领域. 机器学习是一个交叉学 科, 在其发展过程中融合了数学、运筹学、统计学、神经科学、信息论、控制论、计算复杂性理论等学 科的知识. 机器学习简单的说就是让机器进行学习, 学习是核心, 算法是基础, 而机器是算法实现的工 具. Herbert A. Simon 认为所谓学习是指系统中的任何改进, 这种改进使得系统在重复同样的工作或 进行类似的工作时, 能完成的更好. 机器学习则是 “计算机利用经验改善系统自身性能的行为” (Tom M. Mitchell, 1997) ${ }^{[1]}$. 总之, 机器学习就是使用计算机模拟人类的学习活动, 它是研究计算机识别现有 知识、获取新知识、不断改善性能和实现自身完善的方法.

研究表明, 人脑解决问题时首先积累经验, 然后定期 “归纳” 经验, 获得了生活的 “规律”; 当人类 遇到未知的问题或者需要对未来进行 “推测” 时, 人脑使用这些 “规律”, 对未知问题与未来进行 “推 测”, 从而指导自己的生活和工作. 机器学习中的 “训练”, 相当于人类的 “归纳” 过程; 而机器学习中的

\footnotetext{
引用格式: 郭田德, 韩从英, 李明强. 逐层数据再表达的前后端融合学习的理论及其模型和算法. 中国科学: 信息科学, 2019 , 49: 739-759, doi: 10.1360/N112017-00268

Guo T D, Han C Y, Li M Q. Fusion of front-end and back-end learning based on layer-by-layer data re-representation (in Chinese). Sci Sin Inform, 2019, 49: 739-759, doi: 10.1360/N112017-00268
} 
“预测”, 则相当于人类的 “推测” 过程. 所以, 机器学习的处理过程不是因果的逻辑, 而是通过归纳思 想, 得出的相关性结论. 因此, 机器学习的本质是通过利用数据, 训练出模型, 然后使用模型进行预测 的一种方法.

机器学习研究的主要内容包括两个方面. 一个是问题空间如何描述, 即数据的再表达问题, 这是 机器学习面临的首要问题. 其次是推理策略, 根据学习得到的推理策略, 预测未来、指导行动. 为了更 好地理解学习, 需要研究人类是如何实现思考、推理和表达的. 人类的思维和表达离不开语言、文字 和图形等. 人类在思维、归纳和预测过程中, 首先要把所获取的信息进行多次处理, 最终达到记忆和预 测的目的. 用来记忆和预测的信息, 一般都不是数值! 有了计算机这一工具之后, 计算能力得到了大大 的提高, 如何用计算机实现人类对信息的处理过程? 即实现利用计算机对数据的表达、再表达. 该问 题由环境与学习两部分组成, 一是环境提供信息 (数据), 二是学习, 实现数据再表达, 用能够理解、便 于识别的形式记忆下来.

\section{2 学习的二元分层模式}

机器学习的多数研究, 目标主要放在识别或者分类上, 这并不完全正确. 因为最终理想的目标不 仅是为了 “识别率”, 而是为了得到对内容的良好表示. 如果找到了对内容的良好表示, 那么就等于 “理 解”了内容, 因为这些内容可以被用来识别、用来进行语义分析、用来构建抽象逻辑等. 而现在的一 些学习算法的目标 (例如: CNN 等) 却一味地追求识别率 [2]. 因此, 本文提出 “机器学习的二元分层模 式”. 基本的二元学习分层模式是前端学习模式和后端学习模式.

\section{1 机器学习的分类}

机器学习的学习策略现在一般从 3 个方面分类. 第 1 个方面是根据学习所需的推理多少和难易 程度分类 ${ }^{[3]}$; 第 2 个方面是根据学习方式分类; 第 3 个方面是根据学习算法本身分类.

(1) 根据学习所需的推理多少和难易程度分类. 学习策略根据信息转换所需的推理多少和难易程 度, 从简单到复杂可以分为 7 个类型, 即机械学习、示教学习、类比学习、演绎学习、基于解释的学习、 归纳学习和基于神经网络学习.

机械学习是一种单纯依靠记忆学习材料, 而避免去理解其复杂内部和主题推论的学习方法. 在机 械学习系统中, 知识的获取是以较为稳定和直接的方式进行的, 不需要系统进行过多的加工 (再表达). 而对于其他学习系统, 需要对各种建议和训练例子等信息进行加工处理 (再表达) 后, 才能存储起来. 示教学习则是从环境中获取信息, 把知识转换成内部可以使用的表示形式 (数据再表达), 并将新知识 和原有的知识有机地结合为一体. 演绎学习则是推理从公理出发, 经过逻辑变换推导出结论. 演绎推理 的逆过程是归纳推理. 类比学习利用两个不同领域 (源域和目标域) 中的知识相似性, 不是通过建立规 则, 而是通过类比, 从源域的知识推导出目标域的知识, 从而实现学习的目的. 基于解释的学习通过运 用相关领域的知识, 对当前的实例进行分析, 从而构造解释并产生相应知识的一种学习方法, 它需要 首先建立规则. 归纳学习是从大量的经验数据中归纳抽取出一般的判定规则和模式, 是从特殊情况推 导出一般规则的学习方法. 此种学习主要依赖于经验数据, 因此又称为经验学习. 由于归纳依赖于数 据间的相似性, 所以也称为基于相似性的学习. 基于神经网络学习是指模拟人类大脑的神经系统的结 构与功能, 运用大量的处理部件, 采用人工方式构造的一种网络系统. 神经网络的性质主要取决于网络 拓扑结构、网络权重、工作规则. 神经网络的学习问题就是网络权重的调整问题 ${ }^{[4]}$. 人工神经网络是 
机器学习的一个庞大的分支, 现在已有几百种不同的算法 ${ }^{[3]}$.

(2) 根据学习方式分类. 根据数据类型的不同, 对一个问题的建模有不同的方式. 在机器学习或者 人工智能领域, 人们首先会考虑针对不同的学习方式设计各种算法. 在机器学习领域, 有几种主要的 学习方式, 分别是监督学习、无监督学习、半监督学习和强化学习. 监督学习, 是指有结果度量的监督 学习过程. 常见的监督学习算法包括决策树、Boosting 与 Bagging 算法、人工神经网络和支持向量机 等. 无监督学习, 则只能观察特征, 没有结果度量. 常见的无监督学习算法包括聚类分析或关联规则分 析等. 半监督学习, 是指使用大量的未标记数据, 以及同时使用标记数据, 来进行模式识别工作. 使用 半监督学习时, 将会要求尽量少的人员来从事工作, 同时, 又能够带来比较高的准确性. 常见的半监督 学习包括自训练、直推学习、生成式模型等方法 ${ }^{[5]}$. 强化学习, 则不同于连接主义学习中的监督学习, 主要表现在教师信号上, 强化学习中由环境提供的强化信号是对产生动作的好坏作一种评价 (通常为 标量信号), 而不是告诉强化学习系统如何去产生正确的动作.

(3) 根据学习算法本身分类. 根据算法的功能和形式的类似性将算法进行分类, 如基于树的算法 和基于神经网络的算法等. 当然, 机器学习的范围非常庞大, 有些算法很难明确归类到某一类. 而对于 有些分类来说, 同一分类的算法可以针对不同类型的问题.

还有另外一些对机器学习的分类方法. 但是, 几乎现有的机器学习分类的方法都没有考虑、也不 区分机器学习的两个主要研究内容, 即问题空间的描述或者说数据的再表达问题, 以及推理策略问题. 或者将两个内容混为一谈, 或者只是单一的推理策略的学习分类.

\section{2 前端学习与后端学习}

根据对机器学习的两个主要研究内容, 本文提出学习的二元分层模式, 即前端学习模式和后端学 习模式.

学习的首要问题是空间的描述, 即数据的再表达问题. 与之对应, 我们提出前端学习模式, 或者称 为 “特征学习”, 即数据再表达的学习. 这种学习的输入是观测到的原始数据 (样本), 学习的目的是发 现特征, 在有目标类标签 (监督) 或者没有目标类标签 (无监督) 的情况下, 通过学习得到数据的特征; 该层学习的输出是数据 (样本) 的特征, 或者说是对数据的一种再表达. 前端学习主要目的是发现特 征、表达特征, 是数据的重新表达.

现有的前端学习模式可以归纳为如下 3 种:

(1) 专家学习. 根据样本数据的性质和专家的经验, 人工发现和定义特征, 这是前期机器学习重要 的一种特征定义和提取方法, 也是现在一些图像识别系统中常用的方法, 如指纹识别.

(2) 基于数据的前端学习. 根据样本数据, 通过一定的训练算法, 自动学习出特征, 其特点是一般 需要大量的样本数据, 因此也称之为数据驱动的再表达方法.

(3) 基于模型的前端学习. 将问题空间看成是一个函数空间, 通过研究函数空间的性质, 运用数学 的理论和方法, 构造出函数基, 即被认为是数据的特征. 因此, 称之为基于模型驱动的前端学习, 或基 于模型驱动的数据再表达方法.

通过前端学习, 获得了数据的再表达, 即对内容的良好表示. 首先, 可以把这种表示记忆下来. 然 后, 再对这些内容进行分析理解, 构建抽象逻辑, 得到推理策略.

学习的第 2 个研究内容是推理策略, 根据学习得到的推理策略, 预测未来、指导行动. 这个研究内 容一般是在前端学习的基础上的进一步学习, 是前端学习的进一步应用. 与之对应, 我们提出后端学 习模式. 后端学习, 也可以称之为判别学习. 该层学习的输入是前端学习输出的特征, 学习的目的是用 


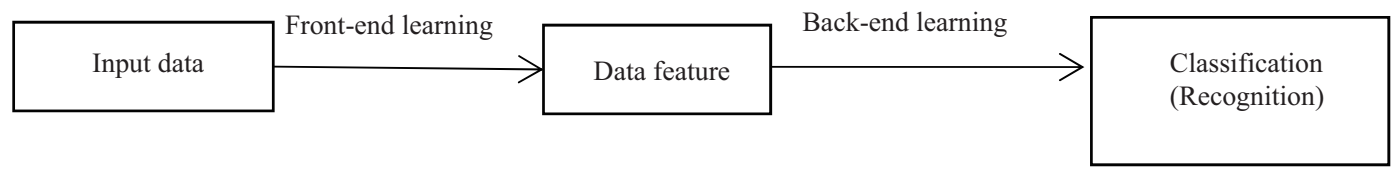

图 1 前后端组合学习

Figure 1 Combination of front-end and back-end learning

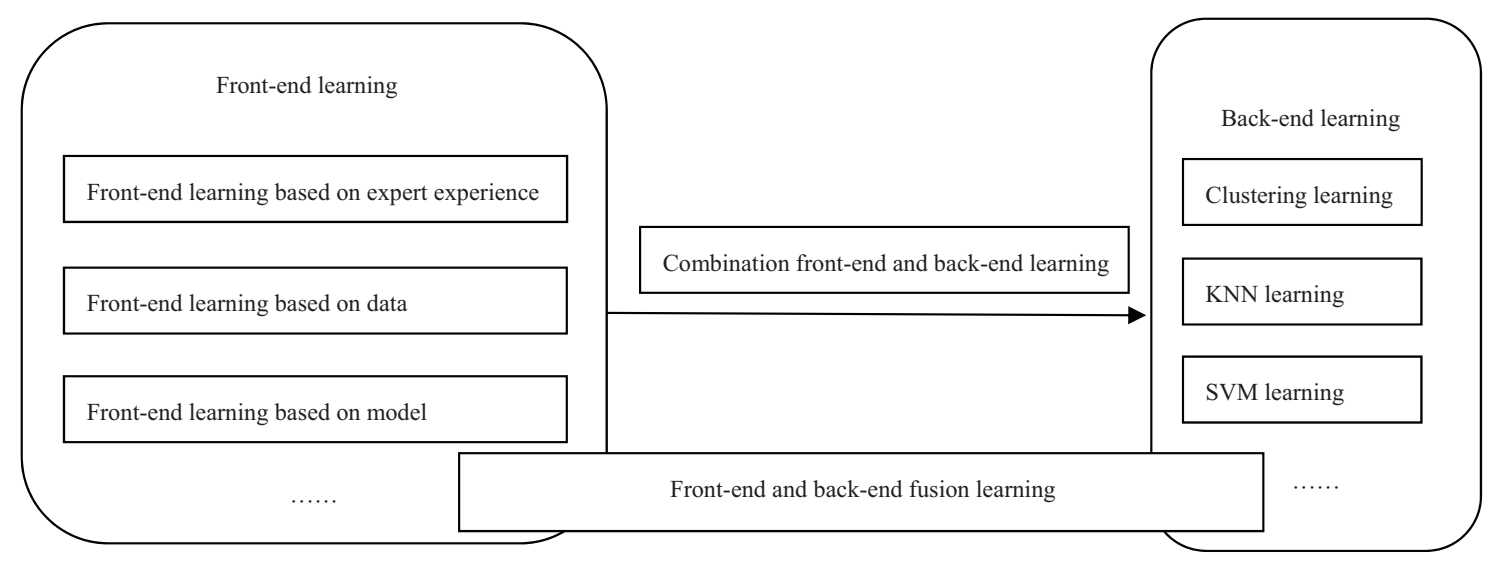

图 2 二元分层学习模式

Figure 2 Two-element layered model of machine learning

于模式分类或者识别, 在有目标类标签 (有监督) 或者没有目标类标签 (无监督) 的情况下, 通过学习 得到模式分类或者识别的能力, 该层学习的输出是对数据 (样本) 的分类或者识别结果.

后端学习的最终目的是对输入数据进行分类或者识别. 前端学习得到特征的目的是为后端学习更 好的记忆、分析、理解、识别或者分类. 前端学习和后端学习可以分别独立进行. 我们可以取一种前 端学习模式得到数据的一种再表达, 即特征; 然后取一种后端学习的模式, 对前端学习得到的特征进 行识别或者分类. 这就是所谓的前后端组合学习, 如图 1 所示.

前后端组合学习是首先定义或者学习得到数据再表达的形式 (特征发现); 然后对输入的数据, 进 行再表达 (特征提取); 最后, 根据数据的再表达, 对原始数据进行识别或者分类 (特征匹配). 这种学 习方式没有将特征发现、特征提取与特征匹配有机地结合在一起. 为此, 我们提出前后端融合学习模 式, 使用统一设计的学习模型, 将前端学习和后端学习融合为一体, 通过学习, 直接输出识别或分类的 结果. 虽然前后端组合学习和前后端融合学习, 输入的都是观测到的原始数据 (样本), 输出的都是对 数据 (样本) 的分类或者识别结果. 但前者是分别选取前端学习模式和后端学习模式, 后者是统一考虑 设计前、后端学习模式.

根据前面定义的学习的二元分层模式, 可以发现, 常见的机器学习算法, 包括聚类算法、最近邻算

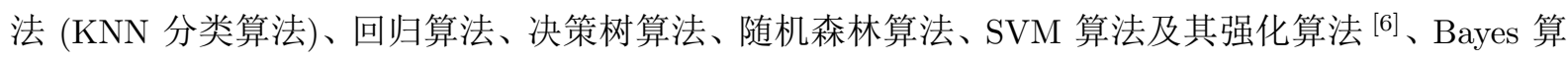
法、EM 算法、极限学习机 (ELM) 算法 ${ }^{[7]}$ 和人工神经网络算法 (包括深度网络) 等 10 个学习算法实 际上不是一个层次的算法. 前 9 个算法实际上都是后端学习算法, 算法并没有涉及数据再表达问题, 而考虑更多的是最后的任务目标. 神经网络学习, 如果后面不接分类器或者识别器, 则属于前端学习的 算法, 但其表示的内容无法解释, 如果接分类器或者识别器则为前后端组合学习或者前后端融合学习 模式. 另外, 我们认为人脑学习应该是前后端融合学习模式, 如图 2 所示. 


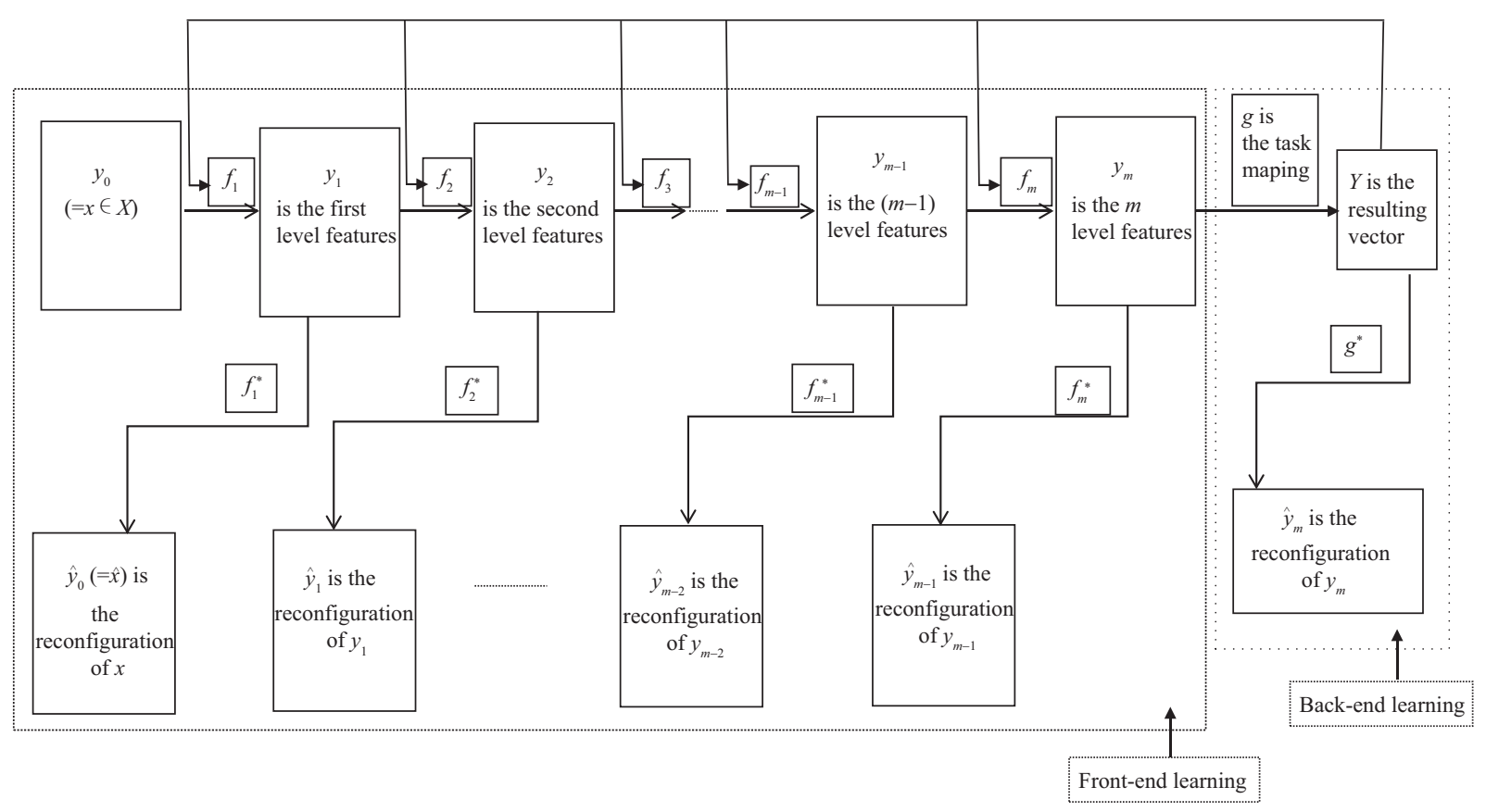

图 3 前后端融合学习框架

Figure 3 Framework of front-end and back-end fusion learning

\section{3 前后端融合学习的框架与最优化模型}

为了将前端学习与后端学习有机地结合在一起, 提高学习的效率和学习精度, 本文建立了前后端融 合学习模型的基本框架, 可以根据基本框架建立优化模型, 设计求解算法. 我们建立了基本框架 (图 3) 和相应的优化模型. 图 3 中, $\left(f_{1}, f_{2}, \ldots, f_{m-1}, f_{m}\right)$ 代表前端学习, $g$ 代表后端学习需要完成任务 (如识 别、分类等) 的映射.

下面给出前端学习中的第 $i$ 级数据再表达的优化模型:

$$
\begin{aligned}
& \max _{f_{i}, f_{i}^{*}}\left\{\sum_{x \in X} \operatorname{Sim}_{i}\left(y_{i-1}, \hat{y}_{i-1}\right)\right\} \\
& \text { s.t. } y_{i}=f_{i}\left(y_{i-1}\right), \quad \hat{y}_{i-1}=f_{i}^{*}\left(y_{i}\right), \quad\left|y_{i}\right|=T_{i} .
\end{aligned}
$$

根据结果向量, 选择重构映射 $g^{*}$, 重构第 $m$ 级特征, 建立后端学习的优化模型:

$$
\begin{aligned}
& \max _{g, g^{*}}\left\{\sum_{x \in X} \operatorname{Sim}_{B}\left(y_{m}, \hat{y}_{m}\right)\right\} \\
& \text { s.t. } \quad Y=g\left(y_{m}\right), \hat{y}_{m}=g^{*}(Y) .
\end{aligned}
$$

如果分别单独调整 $f_{1}, f_{2}, \ldots, f_{m}$ 和 $g$, 则为前后端组合学习, 如果根据结果向量, 再统一调整 
$f_{1}, f_{2}, \ldots, f_{m}$ 和 $g$, 则为前后端融合学习. 建立第 $k(1 \leqslant k \leqslant m)$ 级前后端融合学习的优化模型如下:

$$
\begin{array}{ll}
\max _{\substack{f_{k}, f_{k+1}, \ldots, f_{m}, g, g^{*} \\
f_{k}^{*}, f_{k+1}^{*}, \ldots, f_{m}^{*}}}\left\{\sum_{x \in X}\left(\sum_{i=k}^{m} \operatorname{Sim}_{i}\left(y_{i-1}, \hat{y}_{i-1}\right)+\operatorname{Sim}_{B}\left(y_{m}, \hat{y}_{m}\right)\right)\right\} \\
\text { s.t. } & y_{i}=f_{i}\left(y_{i-1}\right) \quad(i=k, k+1, \ldots, m), \\
& \hat{y}_{i-1}=f_{i}^{*}\left(y_{i}\right) \quad(i=k, k+1, \ldots, m), \\
& \left|y_{i}\right|=T_{i} \quad(i=k, k+1, \ldots, m), \\
& Y=g\left(y_{m}\right), \\
& \hat{y}_{m}=g^{*}(Y) .
\end{array}
$$

上述模型中, $\operatorname{Sim}_{i}$ 表示前端学习中第 $i$ 级特征的重构与第 $i-1$ 级特征的相似度, $\operatorname{Sim}_{B}$ 表示后端学习 中由结果向量重构的特征与前端学习输出的最终特征的相似度, $f_{i}^{*}, g *$ 表示数据的重构函数, $T_{i}$ 指第 $i$ 级特征长度.

\section{4 现有的学习算法归入二元分层模式}

现有的机器学习算法中, 基于前端学习的包括基于专家经验的算法、基于数据驱动的算法和基于 模型驱动的算法. 基于模型驱动的包括函数空间的基分解、非负矩阵分解、主成分分析、数据降维、 稀疏表示、数据压缩和数据低秩矩阵近似 ${ }^{[8]}$ 等. 现有的聚类算法、最近邻算法、回归学习算法、决策 树算法、随机森林算法、SVM 算法、Bayes 算法、EM 算法和人工神经网络算法等 (包括深度网络), 大 多都是后端学习算法, 而人工神经网络算法, 如果是后面不接分类器, 则属于基于数据的前端学习, 如 果接分类器则为前后端组合学习或者前后端融合学习. 另外, 人脑学习是典型的前后端融合学习模式. 基于数据驱动的深度学习网络模拟了人脑的分级工作机制, 可以归入前后端融合学习模式, 最为经典 的是 Hinton 等 ${ }^{[9]}$ 的工作. 深度学习及其各种推广算法在近年被广泛应用于图像、信号和语音等很多 个领域 $[10,11]$, 取得了很好的效果.

\section{3 前端学习—— 数据再表达}

前端学习, 即特征学习. 该层学习的输入是观测到的原始数据 (样本), 学习的目的是发现特征, 在 有或者没有目标类标签的情况下, 通过学习得到数据的特征; 该层学习的输出是数据 (样本) 的特征, 或者说是对数据的一种再表达.

\section{1 大脑对数据的逐层再表达}

为了更好地构建前端学习的模型, 首先介绍大脑的视觉系统. 视觉神经科学围绕大脑的视觉皮层 进行研究, 特别地, 在 1981 年, David Hubel 和 Torsten Wiesel, 以及 Roger Sperry 获得了 Nobel 医学 奖, 这个奖的主要贡献是 “发现了视觉系统的信息处理机制, 可视皮层是分级的”. 此项研究表明人脑 视觉信息的处理是等级制的, 从视网膜上收集到了视觉信号, 由两眼的视觉神经, 传输给视交叉神经, 然后将信号传输到大脑的一个叫外侧膝状体的区域中, 再将视觉信号一层层分门别类, 整理好后传输 到初级视觉皮层. 在视觉皮层之后, 存在不同的通路, 往大脑上方走的通路, 分析的是视觉信号所带来 
的位置信息 (where), 而往下方走的通路, 分析的是何种物体的信息 (what), 也就是用来识别和描述看 到的是什么东西.

心理学家将处在 Brodmann 脑区系统 17 区的皮层简称为 $\mathrm{v} 1$ 皮层 ( $\mathrm{v}$ 代表视觉, 1 代表开始也是 首先的含义). 一般而言, 最初级的信息就是到 v1 这个纹状皮层 (striate cortex). 但是在 v1 之上的其 余不少枕叶皮层也涉及了视觉处理, 比如 $\mathrm{v} 2 \sim \mathrm{v} 4$ 这几个外纹状皮层. 按照现有研究, v1 处理简单视觉, $\mathrm{v} 2$ 是处理图形与物体轮廓 (研究表明空间内大小识别是在 v2 处理的), v3 更多地在传递信息, v4 与颜 色视觉有关. 为什么有这么多皮层? 这个问题涉及到视觉识别的重要研究成果, 即我们在识别视觉信 息的时候有着两个重要通路: 是什么 (what) 和在哪里 (where). 这个发现激发了人们对于神经系统的 进一步思考. 神经 - 中枢 - 大脑的工作过程, 或许是一个不断迭代、不断抽象的过程. 从原始信号, 做 低级抽象, 逐渐向高级抽象迭代. 人类的逻辑思维, 经常使用高度抽象的概念. 例如, 从原始信号摄入 开始 (瞳孔摄入像素), 接着做初步处理 (大脑皮层某些细胞发现边缘和方向), 然后抽象 (大脑判定, 眼 前的物体的形状是圆形的), 然后进一步抽象 (大脑进一步判定该物体是只气球). 这个生理学的发现, 促成了计算机人工智能在 40 年后的突破性发展.

由此可见, 人的视觉系统的信息处理是分级的. 从低级的 $\mathrm{v} 1$ 区提取边缘特征, 再到 v2 区的形状 或者目标的部分等, 再到更高层, 然后是整个目标、目标的行为等. 也就是说高层的特征是低层特征的 组合, 从低层到高层的特征表示越来越抽象, 越来越能表现语义或者意图. 而抽象层面越高, 存在的可 能猜测就越少, 就越利于分类.

所以, 大脑学习的一个重要过程, 就是发现如何对输入的数据逐级重新表达的方法, 以便大脑更 好的记忆、分析、识别或者分类. 机器学习的一个重要过程同样应该是首先学习输入数据的重新表达 方法, 以便更好的存储以及后面的分析、识别或者分类. 数据的再表达可以看成对数据特征的抽取.

特征是人脑学习和机器学习系统的原材料. 特征对机器视觉的最终模型和算法至关重要. 特征就 是对数据的一种重新表达, 或者说是对数据的一种降维表示, 如果数据被很好地表达成了特征, 那么 后端学习就变得非常容易, 通常线性模型就能达到满意的精度. 那么, 学习算法在一个什么粒度上的 特征表示, 才有可能发挥作用? 就一个图片而言, 像素级的特征根本没有价值, 人脑也不可能是对图像 的像素级进行扫描和识别. 如果特征具有结构性 (或者说有含义), 学习算法才能发挥作用.

\section{2 数据与模型混合驱动 —— 机器学习自动获得数据的再表示}

目前, 通过机器学习解决问题的思路 (以视觉感知为例子) 如下: 从开始的通过传感器 (例如 CMOS) 来获得数据, 然后经过预处理、特征提取、特征选择, 再到推理、预测或者识别. 典型的例 子是人工神经网络, 无论是浅层网络还是深度网络, 都需要获得数据, 然后训练数据. 深度学习的每一 层分解的输出是自动获得的特征, 但不完全有直观解释. 这类数据驱动的模型, 模型简单、易于理解. 缺点是需要大量的学习样本; 深度越大, 需要的硬件设备越多, 成本高, 学习时间长; 需要大量的并行 技术; 每一层的输出缺少直观解释. 但是, 似乎人脑学习不需要如此多的样本数据, 能否模拟人脑用少 量的样本得到显著的特征是一个值得研究的课题.

为此, 首先考虑模型驱动的前端学习类型, 包括函数空间的基分解、非负矩阵分解、主成分分析、 数据降维、稀疏表示、数据压缩等. 函数空间的基分解典型的案例是 Fourier 分解、小波分解. 数据的 稀疏表示也是一种很好的前端数据再表达方法. 数学变换追求的所谓稀疏表示, 即如何通过最小数量 的系数尽可能更多地描述信号的能量. 不同类型的信号, 其在不同变换下系数的分布会不同. 信号稀 疏表示的目的就是在给定的超完备字典中用尽可能少的原子来表示信号, 可以获得信号更为简洁的表 示方式, 从而更容易获取信号中所蕴含的信息, 更方便进一步对信号进行加工处理, 如压缩、编码、分 
类、识别等. 信号稀疏表示的两大主要任务就是字典的生成和信号的稀疏分解. 对于字典的选择, 一 般有分析字典和学习字典两大类. 图像数据压缩也是一种典型的原始数据在基下的一种稀疏表示.

数据驱动需要大量样本, 而模型驱动需要的样本量较少, 经验信息丰富, 将二者有机结合起来, 建 立数据与模型混合驱动的逐层数据再表达方法是本文研究的主要思路.

首先, 分析一些典型的数学模型, 如函数空间的基分解. 函数空间中所有函数 (满足一定条件的连 续信号) 都可以在这组基下重新表示, 即在基 (信号的特征) 下的投影. Fourier 级数、小波级数就是对 函数空间中的函数 (连续信号) 在离散 Fourier 基、离散小波基下的重新表示, 从而把连续的时域信号 分解成了频率域信号. 而离散 Fourier 变换、离散小波变换则是离散信号在离散 Fourier 基、离散小波 基下的重新表示, 把采样后的离散时域信号分解成频率域信号. 这类分解可以是在基序列下逐步、逐 层完成, 得到信号的低频部分和高频部分, 分别用来描述信号的概貌和细节. 这些基序列用数学理论 和工具构造, 如 Fourier 基、小波基等. 分解有解析表达式, 每次分解后的数据被直观解释为低频部分 和高频部分, 即对应的信号的概貌和细节. 分解的效果取决于构造的基, 如小波基是通过母小波伸缩、 平移得到, 分解后数据正好适合描述信号的概貌和细节, 往往优于 Fourier 分解, 而且不同的小波基有 不同的效果.

再次, 研究混合模型的构建. 在一个基序列下逐步、逐层分解, 正好与人脑的视觉系统的分级工作 机制相吻合. 基序列用数学理论和工具构造, 如 Fourier 基和小波基或者用某种数学方法构造的基. 基 于数据驱动的深度学习通过每层的训练, 得到每层的参数, 以实现与基序列下逐步、逐层分解, 以及人 脑的视觉系统的分级工作机制相同的效果. 由此, 人脑视觉系统的信息处理、深度学习、数据的基分 解, 都是对数据的重新表达, 可以看成是从数据的特征发现、特征提取到数据降维表达的不同处理方 法. 其最高境界应该是后两者模拟人脑的视觉系统的信息处理机制. 在基序列下逐步、逐层分解, 每一 步、每一层分解后的数据都有直观解释, 与人脑 17 区皮层上的每层都有明确意义相吻合.

人脑的视觉系统是基于学习的, 但似乎并不需要像深度学习那样用如此多的训练样本. 可以将基 序列下的逐步、逐层分解, 人脑的视觉系统的分级工作机制和深度学习结合在一起进行研究, 以提高 深度学习的效率, 使得基序列下逐步、逐层分解和深度学习能够更好地模拟人脑的视觉系统的信息处 理机制. 因此, 本文提出数据与模型混合驱动的逐层数据再表达模型.

基于字典学习的稀疏表示方法 (学习字典) ${ }^{[12]}$ 和基于学习的非负矩阵分解方法 ${ }^{[13]}$ 是本文研究的 两种数据与模型混合驱动的数据再表达模型. 基于字典学习的稀疏表示方法主要解决了指纹压缩问 题. 我们针对指纹图像的特征, 提出了一种基于矩阵稀疏表示的指纹压缩算法. 对指纹图像块构造学 习预定义字典, 对任意给定的新指纹图像, 根据字典序表示出图像的分块, 然后通过最小化 $l_{0}$ 范数, 量 化和编码对指纹图像进行重新表示. 通过考查压缩结果的各项指标, 本文的压缩算法在压缩倍数比较 大时, 比现行的 JPEG, JPEG2000, WSQ 等算法的峰值信噪比有明显的改进, 而且在细节点提取上更 加鲁棒. 基于学习的非负矩阵分解主要解决指纹方向场的提取问题. 首先构建非负矩阵分解模型

$$
\min _{L, B}\|A-L B\|_{F}^{2} \quad \text { s.t. } L \in \mathbb{R}_{+}^{s \times t, r}, \quad B \in \mathbb{R}_{+}^{r, m},
$$

这里 $A=\left[A_{1}, A_{2}, \ldots, A_{m}\right]$, 且 $A_{i} \in \mathbb{R}^{s \times t, 1}, L$ 是基矩阵, 它的每一列表示一幅图像, 称之为基图像, $B=\left[B_{1}, B_{2}, \ldots, B_{m}\right]$ 为系数矩阵. 则对于原始小图像块 $A_{i}$ 由 $L B_{i}$ 表示. 利用基矩阵计算方向场 $Y_{i}=L_{i}^{+} X, X^{\prime}=\left[L_{i} Y_{i}\right]_{+}, L_{i}^{+}$是基矩阵 $L$ 的广义逆, [. $]_{+}$是矩阵元素非负性操作. 然后输入指纹图像 块, 进行训练, 得到矩阵 $L$. 这两种方法都是用较少的训练数据, 训练模型中的某些参数, 如文献 [12] 训练得到的参数是字典, 文献 [13] 训练得到的参数是图像基矩阵. 当然这两种方法只是数据与模型混 合驱动的数据再表达, 并没有体现逐层学习的优势. 


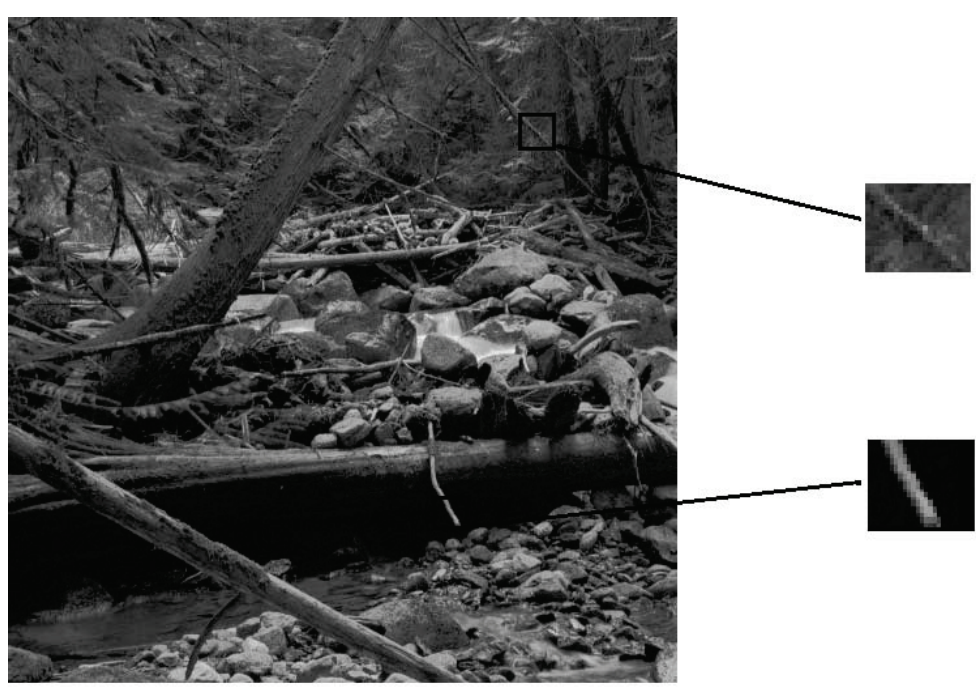

图 4 自然风景图像及其图像块

Figure 4 Natural scenery image and its image block

\section{4 基于数据与模型混合驱动的逐层数据再表达}

图像逐层表达是为了模拟人脑的视觉系统的分级工作机制, 通过逐层表达获得更高层次的信息. 图像逐层表达的主要目的是在每一层的图像中找一组基图像使得对任意给定的该层图像可以由尽可 能少的基图像的组合来表示, 然后将底层基图像进行组合作为高层图像的输入进行训练, 获得更高层 的基图像. 这种逐层表达可以用少量的样本获取好的学习效果, 能很好地克服深度学习需要大样本量 的缺点, 对人类认知功能的模拟更加有效.

数百万年来, 人类的视觉系统在不断处理自然图像环境中进化. 在激烈的生存竞争中, 人类对自 然资源的使用使得人类的视觉系统对自然图像已经有了非常深刻的认知, 并且迅速发展了相应的优化 模型和求解算法 ${ }^{[14,15]}$, 因此我们选择灰度自然风景图像 (图 4) 作为逐层数据再表达的研究对象.

假设一组基图像 1 ) 为 $y_{i} \in \mathbb{R}^{m}, i=1,2, \ldots, n$, 则对任意图像 $x \in \mathbb{R}^{m}$ 的稀疏表示的优化模型为

$$
\min _{\alpha, Y}\|\alpha\|_{0} \quad \text { s.t. } x=Y \alpha,
$$

其中 $Y=\left(y_{1}, y_{2}, \ldots, y_{n}\right) \in \mathbb{R}^{m \times n}, \alpha$ 为系数向量.

在离散的情形下, 给定的一组图像 $x_{i} \in \mathbb{R}^{m}, i=1,2, \ldots, N$, 上述问题改写为

$$
\min _{A, Y}\|A\|_{0} \quad \text { s.t. } X=Y A
$$

其中 $X=\left(x_{1}, x_{2}, \ldots, x_{N}\right) \in \mathbb{R}^{m \times N}, A=\left(\alpha_{1}, \alpha_{2}, \ldots, \alpha_{N}\right),\|A\|_{0}$ 为矩阵零范数 ${ }^{2)}$.

由于上述优化问题为 NP 难的, 压缩感知理论 ${ }^{[16,17]}$ 通常将上述优化问题近似为

$$
\min _{A, Y} F(A, Y ; X)=\|X-Y A\|_{F}^{2}+\lambda \sigma(A) \text { s.t. } X=Y A,
$$

其中 $\sigma(A)$ 为对 $\|A\|_{0}$ 的近似.

1) 本文用列向量来表示灰度图像.

2) 本文中使用的矩阵零范数与 1 范数均表示将矩阵拉成向量对应的定义. 
初始化 $\left(A^{1}, Y^{1}\right)$, 上述优化问题可以通过如下交替极小化算法求解:

$$
\begin{aligned}
A^{k+1} & =\arg \min F\left(A, Y^{k} ; X\right), \\
Y^{k+1} & =\arg \min F\left(A^{k+1}, Y ; X\right) .
\end{aligned}
$$

Olshausen 和 Field ${ }^{[18 \sim 20]}$ 构建模型 (7) 来研究浅层特征表示, 与之不同, 本文选取的 $X$ 不是固定 的, 而是从自然图像中随机抽样, 这种策略使得基图像 $Y$ 鲁棒性更强.

由于复杂的图像块通常是由一些结构化图像块组合而成, 因此当训练样本 $X$ 比较大时, $X$ 的圥 余度很高, 即存在相似度较高的图像块. 使用穴余度高的训练样本会使得训练的针对性不强, 浪费计 算资源以及训练结果偏向于元余度高的训练样本. 我们提出初选图像块的优化模型, 并给出聚类算法 与二部图匹配相结合的近似算法求解初选图像块模型. 另外在传统的稀疏表达算法中, 基图像求解空 间为整个欧氏空间, 本文限定基图像块空间为初选图像块集合. 基于此思想, 我们提出了相应的优化模 型与算法, 使得基图像有更直观的解释 ${ }^{[21]}$.

\section{1 初选图像块的选择模型与算法}

在初始层中, 自然风景图片被分割成小的图像块并作为训练样本. 假定 $x_{i} \in \mathbb{R}^{m}, i=1, \ldots, N$ 为 $N$ 个大小为 $m$ 的训练图像块, $c_{k}(k=1,2, \ldots, K)$ 为从 $N$ 个图像块里选出的 $K$ 个图像块.

本文用聚类方法初选图像块 $c_{k}(k=1, \ldots, K)$. 小图像块聚类的数学模型为

$$
\min _{c_{1}, \ldots, c_{K}} \sum_{i=1}^{N} \min _{k=1, \ldots, K} k\left(x_{i}, c_{k}\right),
$$

这里核函数 $k(x, y)$ 表示两点之间的距离, 且 $k(x, y)=(\phi(x), \phi(y))$, 常用的核函数有欧氏距离核函 数、Gauss 核函数和多项式核函数. 由于

$$
\min \left\{b_{1}, \ldots, b_{K}\right\}=\min \left\{b^{\mathrm{T}} z: z \in \Omega\right\},
$$

其中

$$
\Omega=\left\{Z \in \mathbb{R}^{K \times N} \mid \sum_{k=1}^{K} Z_{k i}=1,1 \leqslant k \leqslant K, 1 \leqslant i \leqslant N\right\} .
$$

故式 (10) 等价于

$$
\min \sum_{i=1}^{N} \sum_{k=1}^{K} Z_{k i} k\left(x_{i}, c_{k}\right) \quad \text { s.t. } z_{i} \in \Omega, i=1, \ldots, N .
$$

令 $X=\left(x_{1}, \ldots, x_{N}\right), C=\left(c_{1}, \ldots, c_{K}\right), K(X, C) \in \mathbb{R}^{N \times K}$ 为 $X$ 与 $C$ 之间的核距离矩阵, 即

$$
K_{i j}(X, C)=k\left(x_{i}, c_{j}\right), 1 \leqslant i \leqslant N, 1 \leqslant j \leqslant K .
$$

模型 (13) 的矩阵形式为

$$
\min G(C, Z)=\operatorname{tr}(K(X, C) Z) \text { s.t. } Z \in \Omega,
$$

我们设计用交替极小化算法求解聚类模型 (14).

传统的聚类算法选择聚类中心的空间为 $\mathbb{R}^{m}$, 而本文选择的聚类中心空间为训练图像块集合 $X$, 即存在矩阵 $Q \in \Delta$ 使得

$$
C=X Q
$$


其中

$$
\Delta=\left\{Q \in \mathbb{R}^{N \times K} \mid Q_{i j} \in\{0,1\}, \sum_{i=1}^{N} Q_{i j}=1, \sum_{j=1}^{K} Q_{i j}=1,1 \leqslant i \leqslant N, 1 \leqslant j \leqslant K\right\} .
$$

将式 (15) 和 (16) 代入 (14) 得

$$
\begin{array}{ll}
\min & F(Q, Z)=\operatorname{tr}(K(X, C) Z) \\
\text { s.t. } & C=X Q, Z \in \Omega, Q \in \Delta .
\end{array}
$$

由于上述优化问题为 0-1 整数规划问题, 求解非常困难. 将其分解为 k-means 聚类问题 (14) 和聚 类中心校正模型:

$$
\min _{Q}\|X Q-C\|_{F}^{2} \quad \text { s.t. } Q \in \Delta .
$$

定义权值矩阵 $W \in \mathbb{R}^{N \times K}$, 其中

$$
W_{i j}=\left\|\phi\left(x_{i}\right)-\phi\left(c_{j}\right)\right\|_{2}^{2}
$$

简单推导可得, 式 (19) 等价于以 $W$ 为权, $X$ 与 $C$ 的最小权完备匹配问题 ${ }^{[22]}$, 即

$$
\min \sum_{i=1}^{N} \sum_{j=1}^{K} Q_{i j} W_{i j} \quad \text { s.t. } Q \in \Delta .
$$

综上所述, 给出 k-means 聚类和二部图匹配结合的算法 1, 求解初选图像块模型 (17).

算法 1 Initial image blocks election algorithm

1: Input $N$ initial image blocks $x_{i}, i=1,2, \ldots, N$ for being trained and choose $K$ image blocks randomly as clustering centers;

2: while it is not convergent do

3: $\quad$ Fix clustering centers $C$, computing (21) for every $i=1,2, \ldots, N$,

$$
Z_{k i}= \begin{cases}1, & \text { if } k=I_{i} \\ 0, & \text { otherwise }\end{cases}
$$

4: $\quad$ Fix $Z$, solving (22) for every $k=1,2, \ldots, K$ :

$$
\sum_{i=1}^{N} Z_{k i} k^{\prime}\left(x_{i}, c_{k}\right)=0
$$

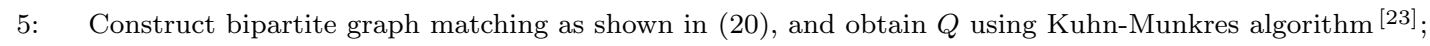

6: $\quad C=X Q$

7: end while

\section{2 基图像选择模型与算法}

下面研究从 $K$ 个初选图像块 $C \in \mathbb{R}^{m \times K}$ 中选择尽量少的基图像块使得对每一个 $c_{k}, k=1, \ldots, K$ 都能够被这些基图像块稀疏表示. 
定义矩阵 $H \in \Delta$ 为选择矩阵, 其中

$$
\Delta=\left\{H \in \mathbb{R}^{K \times K} \mid H_{i j} \in\{0,1\}, \sum_{i=1}^{K} H_{i j} \leqslant 1, \sum_{j=1}^{K} H_{i j} \leqslant 1,1 \leqslant i, j \leqslant K\right\} .
$$

令 $Y \in \mathbb{R}^{m \times K}$ 为基图像矩阵, 即

$$
Y=\left(y_{1}, \ldots, y_{K}\right)=\left(c_{1}, \ldots, c_{N}\right)\left(\begin{array}{ccc}
H_{11} & \ldots & H_{1 K} \\
\vdots & & \vdots \\
H_{K 1} & \ldots & H_{K K}
\end{array}\right)
$$

若 $H_{i j}=1$ 则表示第 $j$ 个基图像 $y_{j}$ 来自第 $i$ 个初选图像块 $x_{i}$; 若 $H_{i j}=0$ 对任意的 $i=1, \ldots, K$ 都 成立则表示第 $j$ 个基图像 $y_{j}$ 为 0 .

基图像块 $Y$ 稀疏表示初选图像块 $C$ 的优化模型为

$$
\min _{H, A} F(H, A)=\frac{1}{2}\|C-Y A\|_{F}^{2}+\lambda_{1} \sigma(A) \quad \text { s.t. } Y=C H, H \in \Delta,
$$

其中 $A$ 为系数矩阵, $\sigma(A)$ 表示 $A$ 的稀疏性函数.

令 $s$ 为选择的基图像块个数, 则

$$
s=\sum_{i=1}^{K} \sum_{j=1}^{K} H_{i j} .
$$

为保证基图像个数尽量少, 将 $s$ 作为正则项加入模型 (25) 即

$$
\begin{array}{ll}
\min _{H, A} & F(H, A)=\frac{1}{2}\|C-C H A\|_{F}^{2}+\lambda_{1} \sigma(A)+\lambda_{2} \sum_{i=1}^{K} \sum_{j=1}^{K} H_{i j} \\
\text { s.t. } & H_{i j} \in\{0,1\}, 1 \leqslant i, j \leqslant K, \\
& \sum_{i=1}^{K} H_{i j} \leqslant 1,1 \leqslant j \leqslant K, \\
& \sum_{j=1}^{K} H_{i j} \leqslant 1,1 \leqslant i \leqslant K .
\end{array}
$$

由于 $H_{i j} \in\{0,1\}$ 使得模型 $(26)$ 求解非常困难, 将 $H_{i j}$ 松他为取值在 $[0,1]$ 上的连续变量, 将上 述优化问题变成连续优化问题

$$
\begin{array}{ll}
\min _{H, A} & F(H, A)=\frac{1}{2}\|C-C H A\|_{F}^{2}+\lambda_{1} \sigma(A)+\lambda_{2} \sum_{i=1}^{K} \sum_{j=1}^{K} H_{i j} \\
\text { s.t. } & 0 \leqslant H_{i j} \leqslant 1, \quad 1 \leqslant i, j \leqslant K, \\
& \sum_{i=1}^{K} H_{i j} \leqslant 1, \quad 1 \leqslant j \leqslant K, \\
& \sum_{j=1}^{K} H_{i j} \leqslant 1,1 \leqslant i \leqslant K .
\end{array}
$$


下面给出求解问题 (27) 的方法.

当 $H$ 固定时, 式 (27) 关于 $A$ 的极小化模型为

$$
\min _{A} R(A)=\frac{1}{2}\|C-C H A\|_{F}^{2}+\lambda_{1} \sigma(A) .
$$

定义算子 $T_{\sigma}: \mathbb{R}^{K \times K} \rightarrow R$,

$$
T_{\sigma, \lambda}(Q)=\arg \min _{A \in \mathbb{R}^{K \times K}} \frac{1}{2}\|A-Q\|_{F}^{2}+\lambda \sigma(A) .
$$

当 $\sigma(A)$ 取特殊函数时, 式 (29) 存在解析解, 我们采用 FISTA 算法 (算法 2) 求解模型 (28).

\section{算法 2 FISTA algorithm}

1: Input elected image matrix $C$, and the Lipschitz continuous constant $L$ of $\nabla R(A)$;

2: Choose initial point $A_{0}$, set $B_{1}=A_{0}, t_{1}=1, k=1$;

3: while it is not convergent do

4:

$$
\begin{aligned}
& A_{k}=T_{\sigma, \lambda}\left(B_{k}-\frac{1}{L} \nabla R\left(B_{k}\right)\right), \\
& t_{k+1}=\frac{1}{2}\left(1+\sqrt{1+4 t_{k}^{2}}\right), \\
& B_{k+1}=B_{k}+\frac{t_{k}-1}{t_{k+1}}\left(A_{k}-A_{k-1}\right) ;
\end{aligned}
$$

5: end while

当 $A$ 固定时,式 (27) 关于 $H$ 的模型变为带有线性不等式约束和界约束的二次规划问题, 本文采 用有效集算法 ${ }^{[24]}$ 进行求解. 由于有效集算法求解得到的 $H$ 为连续矩阵 $H \in[0,1]$, 故本文采用贪婪 的思想进行矩阵二值化, 参见算法 3 .

\section{算法 3 Matrix binarization algorithm}

1: Input continuous matrix $H \in[0,1]$ and the threshold $\epsilon \in[0,1]$;

2: Sort all the elements of $H$ from large to small, denote the sorting sequence pair as $\left(p_{i}, q_{i}\right), i=1, \ldots, K^{2}$;

3: for $i=1, \ldots, K^{2}$ do

4: $\quad$ if $H\left(p_{i}, q_{i}\right) \geqslant \epsilon$

$H\left(p_{i},:\right)=0, H\left(:, q_{i}\right)=0, H\left(p_{i}, q_{i}\right)=1 ;$

else $H\left(p_{i}, q_{i}\right)=0$

5: end for

算法 3 中对 $H$ 排序的目的在于优先考虑较大元素变为 1 , 步骤 4 是为了保证不同行不同列中只 有一个元素为 1 , 即保证约束 $(27 \mathrm{~b}) \sim(27 \mathrm{~d})$ 的成立.

综上所述, 给出选择基图像块的完整算法 4.

假设 $y_{i} \in \mathbb{R}^{m}, i=1, \ldots, s$ 为从初选图像块里选择的 $s$ 个基图像块, 则对任意给定的 $x \in \mathbb{R}^{m}$ 图像 块, 我们希望可以用基图像块稀疏表示原始图像块 $x$. 求解

$$
\min _{\alpha} \frac{1}{2}\left\|\sum_{i=1}^{s} \alpha_{i} y_{i}-x\right\|_{2}^{2}+\lambda\|\alpha\|_{1}
$$




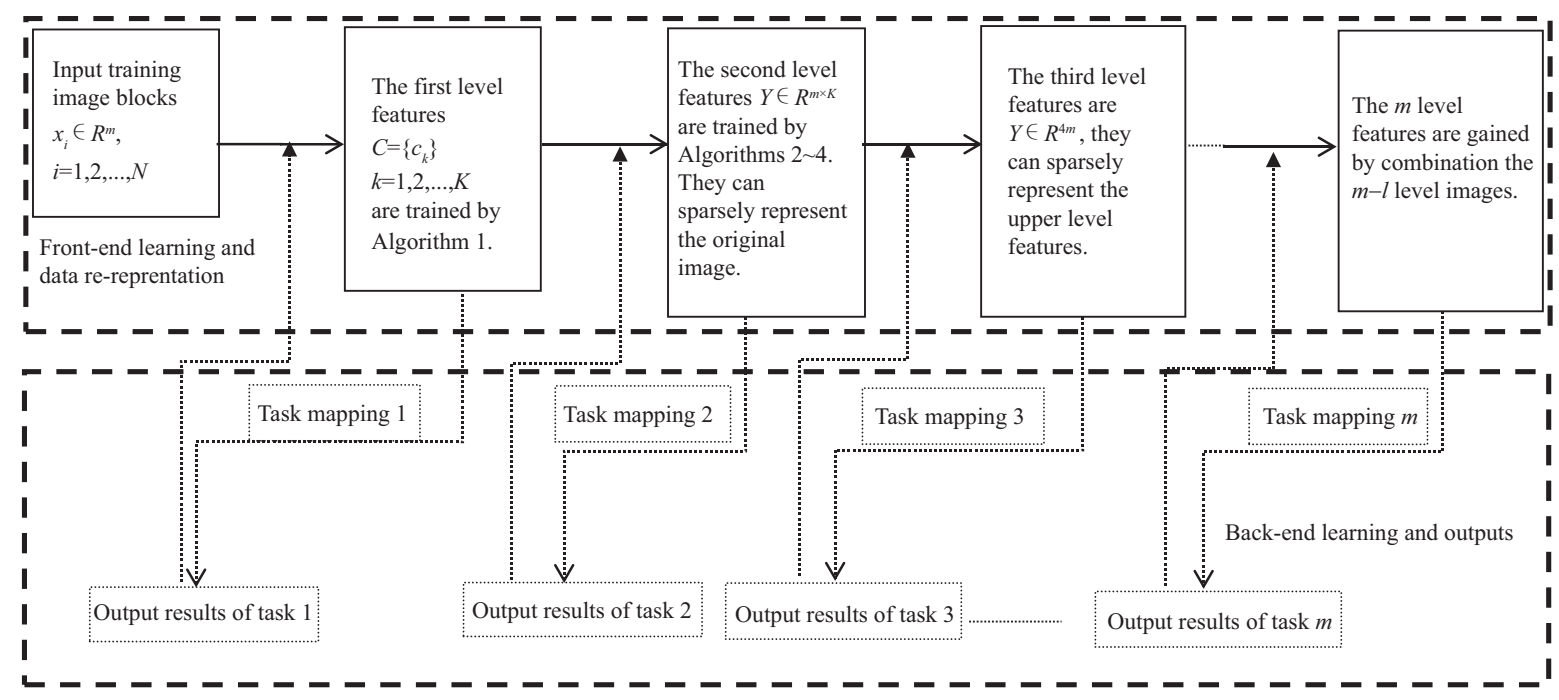

图 5 逐层数据再表达算法流程

Figure 5 Framework of data re-representation method of layer by layer

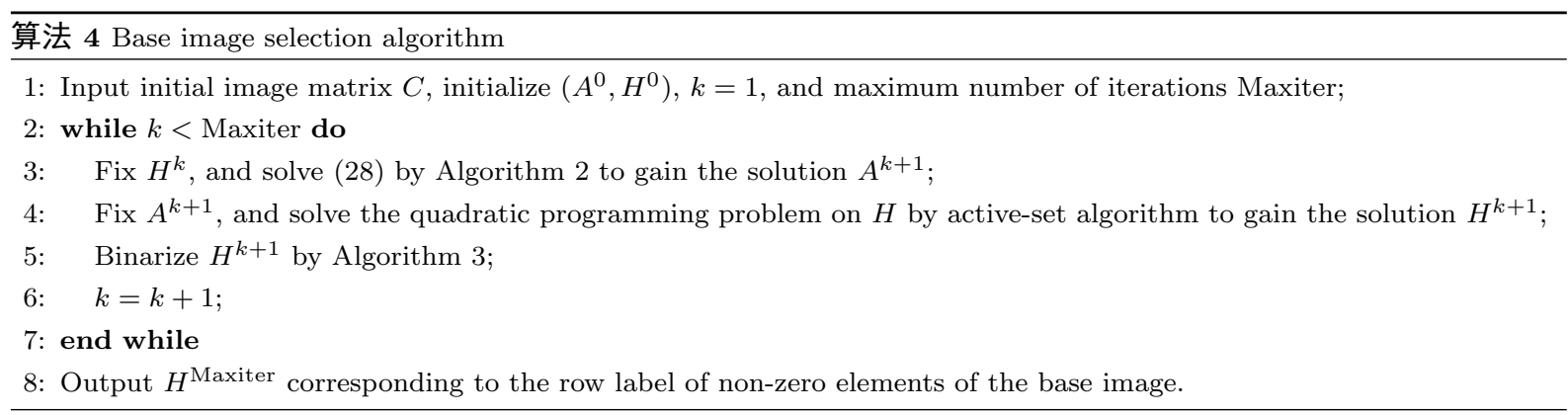

的解 $\alpha^{*}$, 则重建图像 $x^{*}=\sum_{i=1}^{s} \alpha_{i}^{*} y_{i}$.

若下一层图像空间为 $\mathbb{R}^{4 m}$. 为构建下一层图像稀疏表达的训练图像, 需要将底层基图像重新组合 得到高层的训练图像. 将所有的 $y_{i} \in \mathbb{R}^{m}$ 进行组合可以得到 $\mathbb{R}^{4 m}$ 的训练图像集合, 将此集合作为下一 次稀疏表达的输入即可.

由图 5, 将图像视觉的逐层表达的完整流程描述出来. 在本流程中, 通过前端学习抽象出了不同粒 度的特征, 所以可以根据特征粒度给出适合本特征的任务, 例如根据不同的特征可以设计不同的任务, 例如粗分类、细分类、识别等进行后端学习, 学习之后输出结果; 反之, 也可以根据不同的任务结果情 况调整特征粒度. 由于我们选用的是模型与数据混合驱动, 因此特征有很好的解释性, 任意层的特征可 以完成某项特定的任务, 可以选择任务也可以不选择任务, 此部分内容是后面着重研究的内容.

\section{3 数值实验}

本文选择 10 幅 $512 \times 512$ 的自然风景图片 ${ }^{3)}$ 作为研究对象, 如图 6 所示. 首先将自然图像切成 $16 \times 16$ 的小块. 实验选择切割得到的 10240 个小图像块作为初始层的训练集. 实验环境为 Windows7Intel I7 处理器- MATLAB 2014. 下面分别给出初始层中初选图像块以及基图像选择的数值结果.

3) http://redwood.berkeley.edu/bruno/sparsepyr/. 

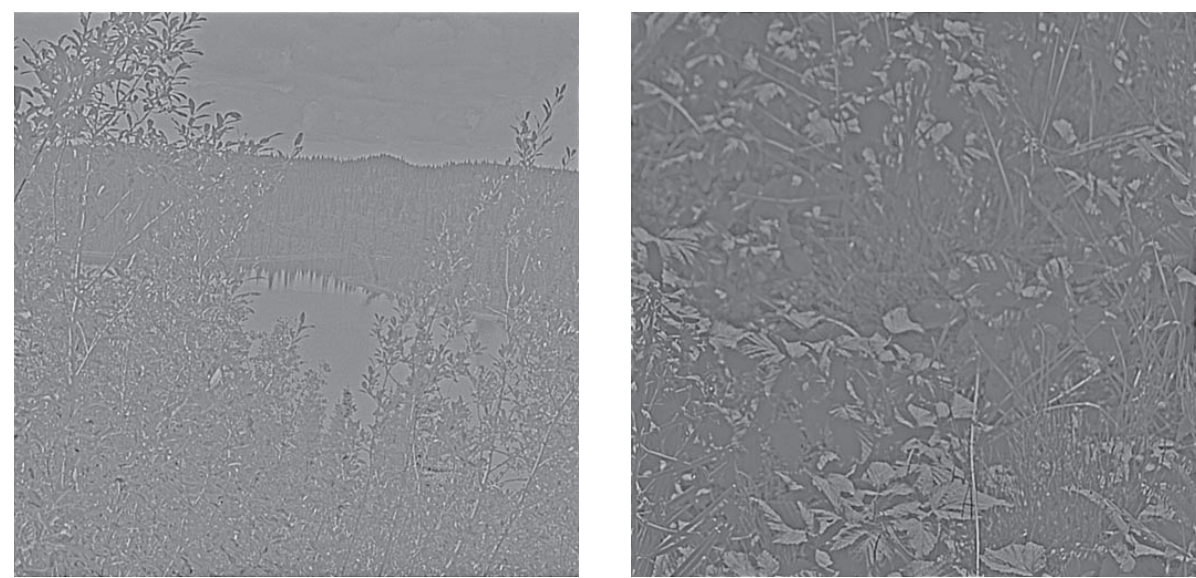

图 6 两幅自然风景测试图像

Figure 6 Two natural landscape test images

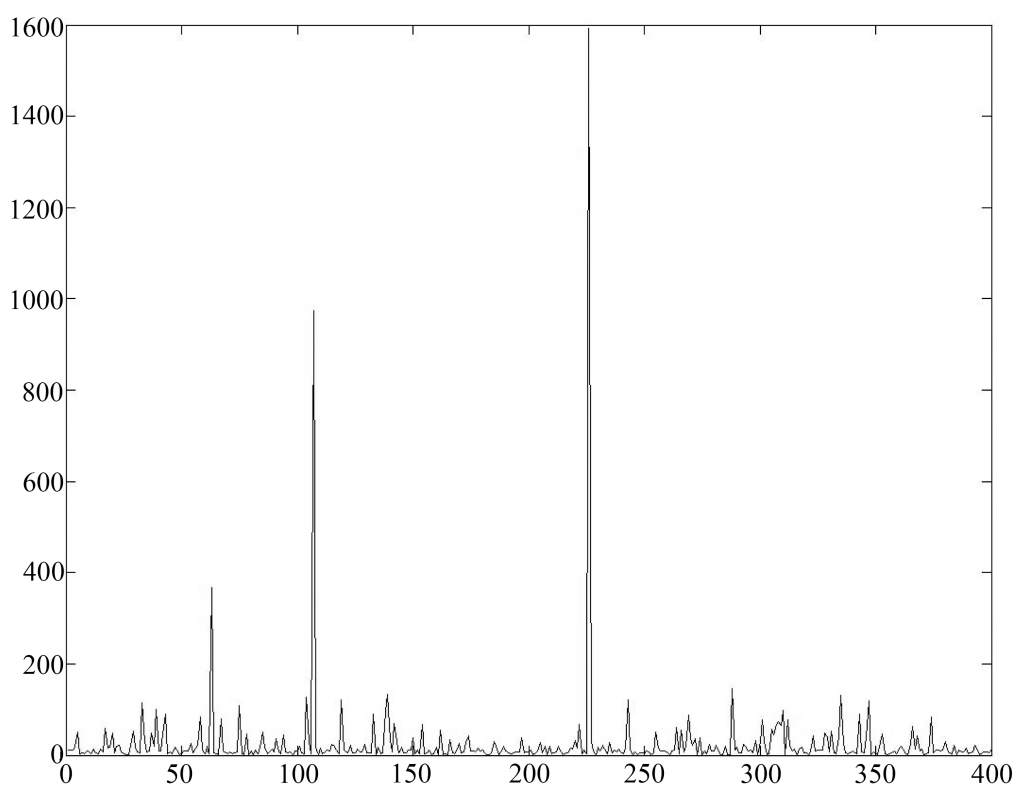

图 7 每一类元素个数分布图

Figure 7 Number distribution diagram of each kind of element

\subsection{1 初选图像块选择数值结果}

初选图像块算法中选择 $K=400$, 选择欧氏距离作为相似度度量方式.

图 7 描述了属于初选图像块所在类的元素个数分布图, 横轴表示图像块的类, 第 50 类, 第 100 类, ..., 共 400 类; 纵轴表示在当前类中图像块的数量. 由图 7 可以看出每一类中元素的个数均不相同, 第 226 类元素个数最多, 超过 1500 个, 其次是第 107 类元素个数接近 1000 , 剩余的大部分类别中元素个 数均小于 200 .

图 8 描述了第 226 类中的部分元素, 此类元素没有明显的结构性信息, 相对较为平滑, 非常类似 于自然风景图像中的背景信息. 通常来说, 背景信息在自然图像中是非常常见的, 因此聚类结果中此 

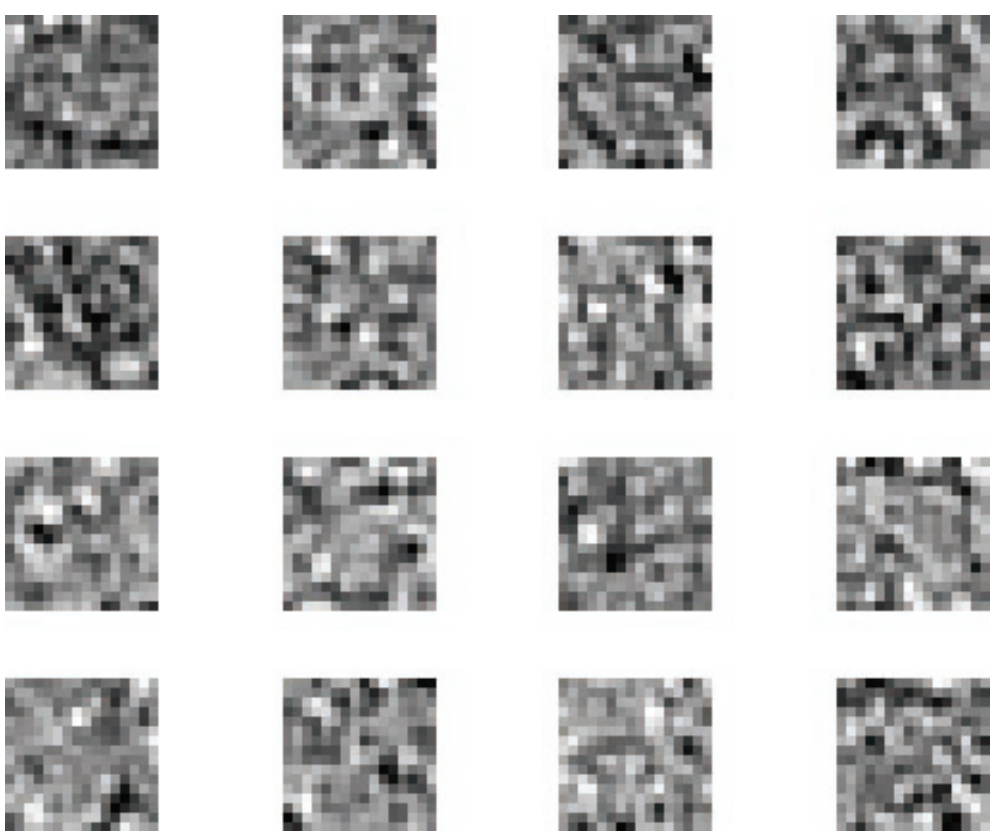

图 8 第 226 类部分元素

Figure 8 Partial elements of the 262th kinds
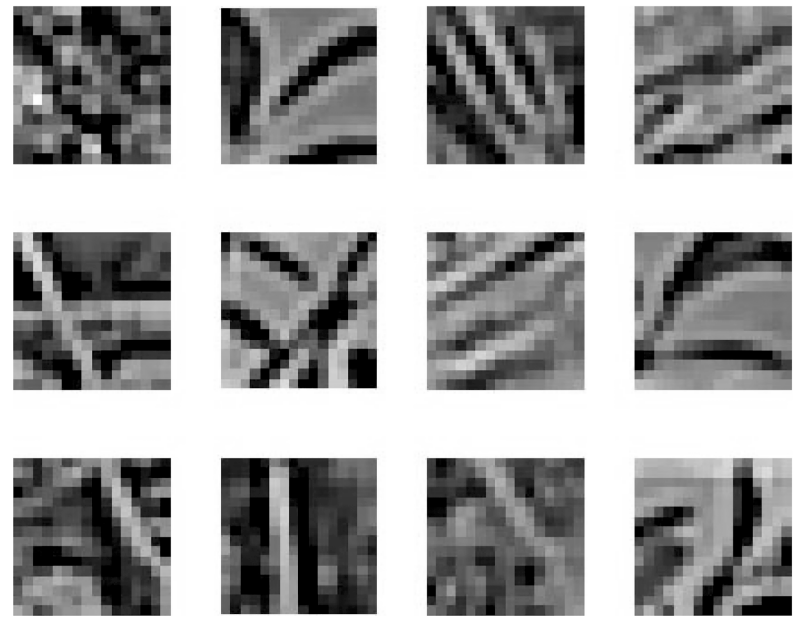

图 9 部分初选图像块

Figure 9 Partial primary image blocks

类元素的个数最多是正常现象.

图 9 给出部分初选图像块, 图中初选图像块差异较大, 第 1 幅初选图像块为纹理信息不明显的背 景图像块, 剩余的图像块纹理信息明显, 且差异性较大, 达到了设计初选图像块算法的期望.

\subsection{2 基图像块选择数值结果}

求解基图像块选择模型 (26), 选择正则化参数 $\left(\lambda_{1}, \lambda_{2}\right)=(0.01,0.01)$, 系数稀疏函数 $\sigma(A)=\|A\|_{1}$. 由 FISTA 算法的收玫性条件知, 本文采用回朔策略选择 Lipschitz 连续常数 $L$ (具体参看文献 $[25$, 


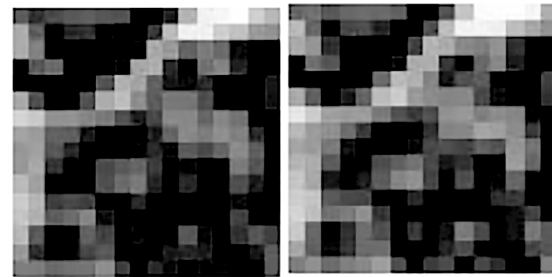

(a)

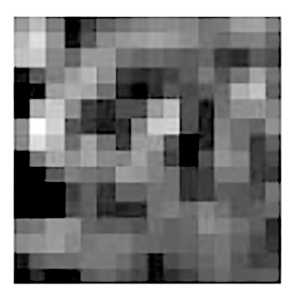

(c)

$\mathrm{PSNR}=27.01$

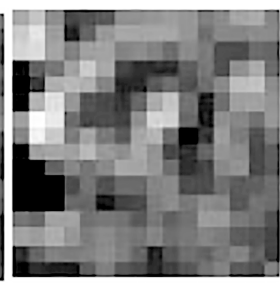

$\mathrm{PSNR}=26.81$
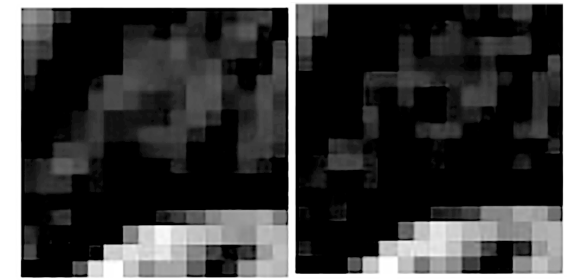

(b)

PSNR $=26.98$

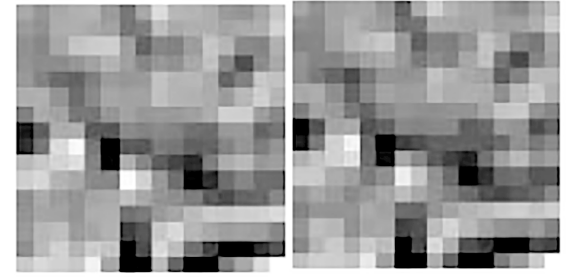

(d)

$\mathrm{PSNR}=27.45$

图 10 初始层基图像重建结果

Figure 10 Base-image reconstruction results of initial layer. The left image blocks of (a) (d) are $16 \times 16$ original image blocks randomly selected; the right image blocks of $(\mathrm{a}) \sim(\mathrm{d})$ are the results of sparse reconstruction of the corresponding left image blocks

引理2.3]). 在矩阵二值化算法中, 取 $\epsilon=0.124$. 算法得到的基图像块数目为 150 .

图 10(a) (d) 中, 左侧为随机选取的 $16 \times 16$ 大小的自然图像块, 右侧用选取的基图像块稀疏复原 结果及其 PSNR 值. 图中左侧原始图像跟右侧重建图像的差别不是很大, 仅有略微不同, 由此可见初 始层图像可以由得到的基图像的线性组合来表示.

图 11 分别显示图 10(a) (d) 4 幅图像稀疏系数的分布情况. 横轴表示每个基图像块的索引, 共有 150 个基图像块, 1 到 150; 纵轴表示用基图像块的线性组合表示原图像, 每一个基图像块的贡献程度 或稀疏系数. 由图 11 可以看出, 大部分稀疏系数在 0 附近跳跃, 存在极少的系数有很大的跳跃, 这说 明算法在实现重建的图像中仅有极少数的基图像起决定性作用.

给定原始图像 $x \in \mathbb{R}^{256}$, 稀疏系数 $\alpha \in \mathbb{R}^{150}$ 和阈值 $\eta$, 定义 $\beta(\eta)$ :

$$
\beta(\eta)_{i}= \begin{cases}0, & \left|\alpha_{i}\right|<\eta, \\ \alpha_{i}, & \left|\alpha_{i}\right| \geqslant \eta .\end{cases}
$$

在 $\beta(\eta)$ 下重建的图像为

$$
x(\eta)=\sum_{i=1}^{150} \beta(\eta)_{i} y_{i} .
$$

定义稀疏程度

$$
\operatorname{Spare}(\eta)=\frac{150-\|\beta(\eta)\|_{0}}{150} .
$$

图 12 和 13 分别描述了不同稀疏程度下重建的图像块与完整的图像. 当稀疏程度较低时, 重建图 像的质量下降并不明显, 甚至图像块在稀疏度为 0.2 时, 重建图像块质量比稀疏度 0.1 时还高. 但随着 稀疏程度逐渐提高时, 重建图像质量变低. 特别是当 Spare 取 0.4 时, 重建图像块失真严重, 而且重建 的完整图像看起来过于光滑, 丢失了大量的纹理信息, 这说明选取的基图像的代表性仍然有欠缺, 还 存在一定的乤余程度. 

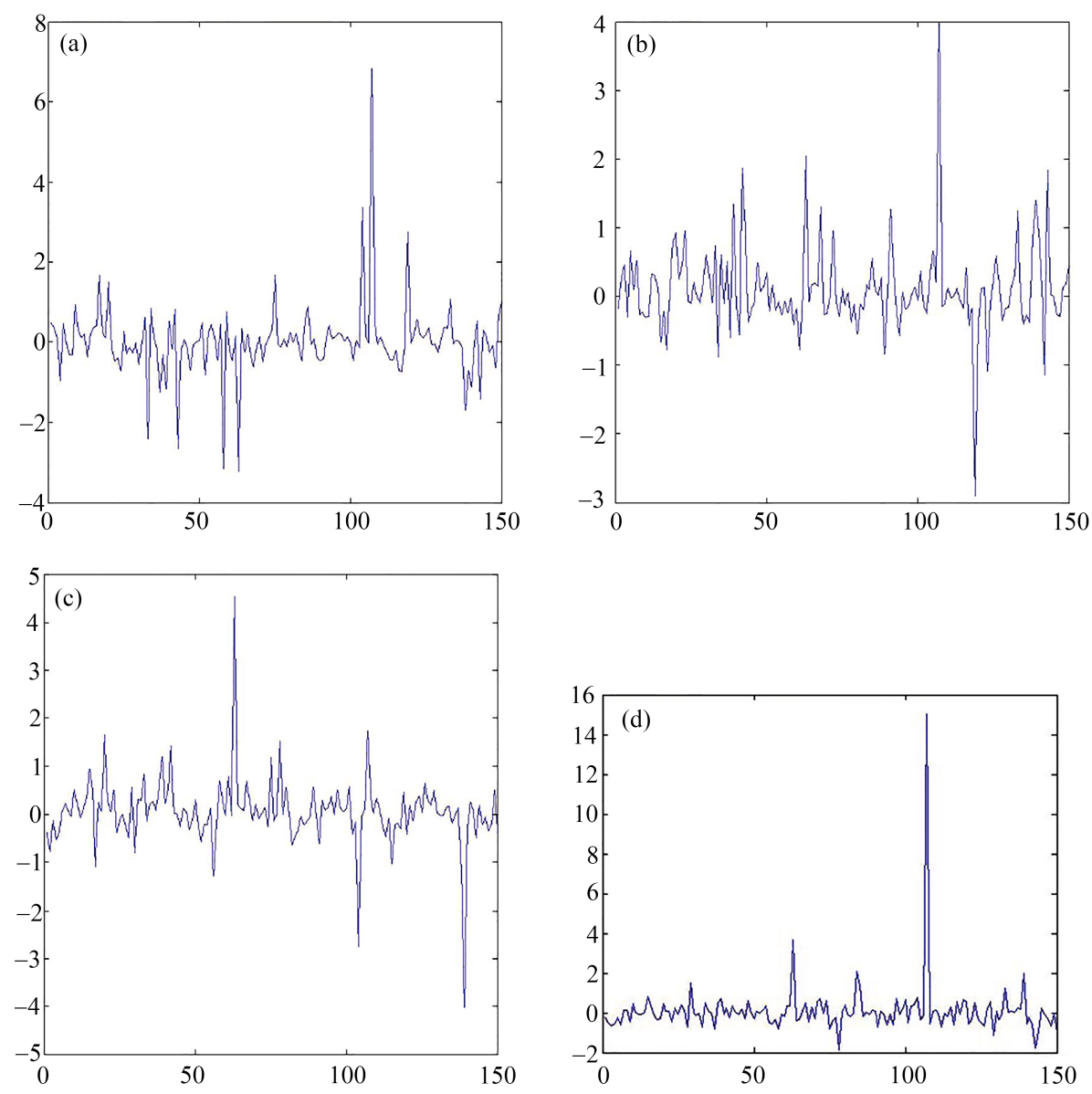

图 11 (网络版彩图) 基图像稀疏系数分布

Figure 11 (Color online) Sparse coefficient distribution of base images. (a) (d) are the sparse coefficient distribution of corresponding images $(\mathrm{a}) \sim(\mathrm{d})$ of Figure 10 expressed by base images, respectively

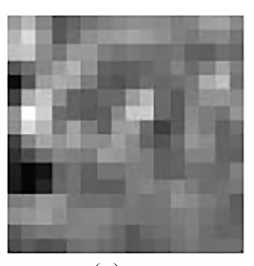

(a)

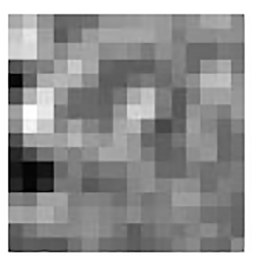

(b)

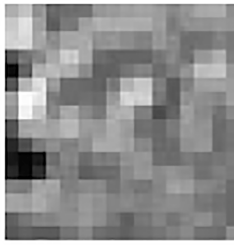

(c)

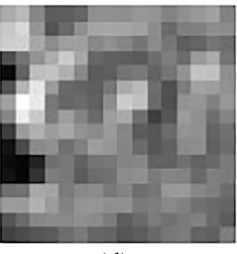

(d)

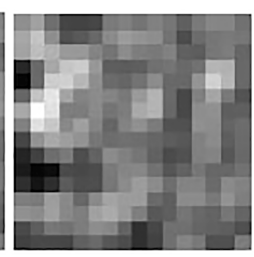

(e)

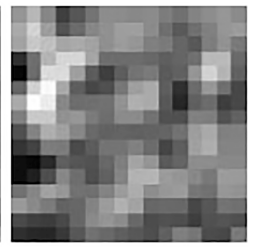

(f)

图 12 不同的稀疏程度下重建的图像块

Figure 12 Reconstructed image blocks with different sparsity. (a) Original image block; (b) Spare=0, PSNR=26.80; (c) Spare=0.1, PSNR=25.29; (d) Spare=0.2, PSNR=26.64; (e) Spare=0.3, PSNR=22.02; (f) Spare=0.4, PSNR=16.04

\subsection{3 实验启示}

本文构建的图像逐层稀疏表达与算法包括两个步骤: 初选图像块的模型与算法; 基图像块选择的 模型与算法. 初选图像块模拟大脑视觉系统中的输入层, 基图像块选择模拟了视觉系统的 v1 层. 在每 一层中均给出了具体的优化模型和算法. 初选图像块模型采用聚类算法与二部图匹配相结合的近似算 


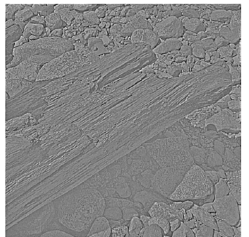

(a)

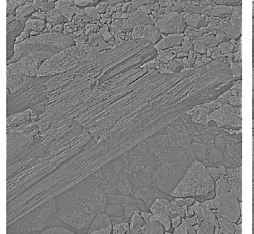

(b)

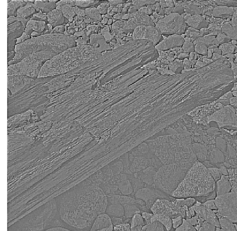

(c)

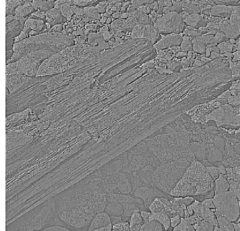

(d)

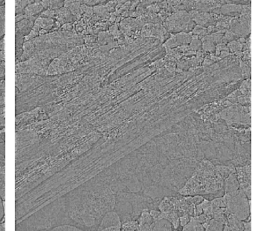

(e)

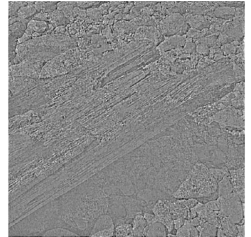

(f)

图 13 不同的稀疏程度下重建的完整图像

Figure 13 Reconstructed images with different sparsity. (a) Original image; (b) Spare=0, PSNR=28.20; (c) Spare=0.1, PSNR=30.03; (d) Spare=0.2, PSNR=29.15; (e) Spare=0.3, PSNR=24.83; (f) Spare=0.4, PSNR=23.14

法求解. 本文限定基图像块的选择空间为初选图像块集合, 并提出了相应的模型与算法, 使得基图像 有更直观的解释. 此设计完全符合第 2,3 节提出的理论思想, 利用数学模型加少量的图像样本进行训 练, 每层都进行重构操作, 对比重构结果, 指导训练参数的调整. 数值结果表明初选图像块相似性较小, 且具有一定的代表性, 同时在较低的稀疏程度下基图像块基本上可以重建初始层的图像块, 但当稀疏 程度较高时重建效果并不十分理想, 需要进一步研究.

本文提出的图像分层表达理论模型和算法设计开创了描述图像特征的新思路, 完全用小样本模拟 人类视觉系统, 而且使得每一层上的表示有了直观的解释, 改变了深度学习中必须构造和选取大量样 本的情况. 我们此类模型的设计及实验效果表明这种分层表达的思路是可行的, 随着更深入的研究, 调整对初选图像块和基图像块的选择策略, 结果会有更好的改善, 即在初始层上将可以实现稀疏度高 的图像的理想表达, 从而模拟人脑的更高层上的图像抽象.

\section{5 总结}

根据机器学习的两个主要研究内容, 本文将学习分成了两层, 即前端学习和后端学习; 将现有的学 习算法在二元分层模式下, 重新进行了归类; 提出了前后端组合学习和前后端融合学习的概念, 并构 建了前后端融合学习的理论框架和优化模型; 针对前端学习, 模拟大脑的分级工作机制, 结合大脑学习 的经验积累和联想、只需要少量样本等特点, 提出了数据与模型混合驱动的逐层数据再表达的构建方 法; 以视觉 (图像) 学习为例, 给出了一种数据与模型混合驱动的前后端融合的逐层数据再表达的具体 方法, 该方法在学习时利用优化模型寻找初始图像块, 使得小样本的图像块有充分地表示意义, 这可 以看做大脑对眼睛摄入的图像进行初始处理后输入到大脑皮层 v1 层的信息. 然后构建学习模型, 训 练计算基图像, 这相当于模拟大脑皮层 v1 层的功能, 对图像进一步抽象, 提取初始特征, 是对数据的 第 1 级再表达. 我们再利用第 1 级基图像 (特征), 构造不同的组合模型, 得到各种不同的图像认识, 这 是大脑的高层抽象, 从而进一步到达 v2, v3 层甚至更高层等. 这样, 可以通过建立逐级优化模型, 实现 数据与模型混合驱动的逐层数据的再表达, 更好地模拟大脑逐级工作机制, 给出数据的一种良好表示.

本文针对学习的二元分层模式中的前端学习, 模拟人脑逐级工作机制, 初步建立了数据与模型混 合驱动的逐层数据再表达中第 1,2 层的优化模型, 设计了求解算法. 实验结果表明, 第 1 层的优化模 型和算法基本能够模拟人脑 $\mathrm{v} 1$ 区的工作机制. 但是第 2 层乃至更高层的优化模型和算法还没有达到 预期的效果, 有待于进一步完善. 现有的机器学习算法中的多数算法属于后端学习算法, 前端学习算 法意义重大但成果较少. 另外, 前后端融合学习的理论、结构、模型和算法也需要更多的探讨. 


\section{参考文献}

1 Mitchell T M. Machine Learning. New York: McGraw-Hill Science, 1997

2 Sabour S, Frosst N, Hinton G E. Dynamic routing between capsules. In: Proceedings of the 31st Conference on Neural Information Processing System, Long Beac, 2017

3 Xie J B, Xing J L, Zhang L N, et al. 20 Lectures on Visual Machine Learning. Beijing: Tsinghua University Press, 2015 [谢剑斌, 兴军亮, 张立宁, 等. 视觉机器学习 20 讲. 北京: 清华大学出版社, 2015]

$4 \mathrm{Wu}$ W, Yang J. $L_{1 / 2}$ regularization methods for weights sparsification of neural networks. Sci Sin Math, 2015, 45: 1487-1504 [吴微, 杨洁. 用于神经网络权值稀疏化的 $L_{1 / 2}$ 正则化方法. 中国科学 数学, 2015, 45: 1487-1504]

5 Liu J W, Liu Y, Luo X L. Semi-supervised learning methods. Chinese J Comput, 2015, 38: 1592-1617 [刘建伟, 刘媛, 罗雄麟. 半监督学习方法. 计算机学报, 2015, 38: 1592-1617]

6 Ma L R, Song D D, Liao L J, et al. PSVM: a preference-enhanced SVM model using preference data for classification. Sci China Inf Sci, 2017, 60: 122103

7 Deng C W, Huang G B, Xu J, et al. Extreme learning machines: new trends and applications. Sci China Inf Sci, 2015, 58: 020301

8 Feng X D, He X M. Robust low-rank data matrix approximations. Sci China Math, 2017, 60: 189-200

9 Hinton G E, Salakhutdinov R R. Reducing the dimensionality of data with neural networks. Science, 2006, 313: $504-507$

$10 \mathrm{Qu} \mathrm{W}$, Wang D L, Feng S, et al. A novel cross-modal hashing algorithm based on multimodal deep learning. Sci China Inf Sci, 2017, 60: 092104

11 Gao W, Zhou Z H. Dropout rademacher complexity of deep neural networks. Sci China Inf Sci, 2016, 59: 072104

12 Shao G Q, Wu Y P, Yong A, et al. Fingerprint compression based on sparse representation. IEEE Trans Image Process, 2014, 23: 489-501

13 Shao G Q, Han C Y, Guo T D, et al. An NMF-based method for the fingerprint orientation field estimation. In: Proceedings of Computer and Information Science, Warsaw, 2012. 93-104

14 Li M Q, Han C Y, Guo T D. New gradient algorithms for optimization problems constrained by a cartesian product of unit balls. Acta Math Appl Sin, 2018, 41: 43-54 [李明强, 韩从英, 郭田德. 新的梯度算法求解单位球笛卡尔积约 束优化问题. 应用数学学报, 2018, 41: 43-54]

15 Li M Q, Han C Y, Wang R X, et al. Shrinking gradient descent algorithms for total variation regularized image denoising. Comput Opt Appl, 2017, 68: 643-660

16 Donoho D L. Compressed sensing. IEEE Trans Inf Theory, 2006, 52: 1289-1306

17 Candes E J, Romberg J, Tao T. Robust uncertainty principles: exact signal reconstruction from highly incomplete frequency information. IEEE Trans Inf Theory, 2006, 52: 489-509

18 Olshausen B A, Field D J. Emergence of simple-cell receptive field properties by learning a sparse code for natural images. Nature, 1996, 381: 607-609

19 Olshausen B A, Field D J. Sparse coding with an overcomplete basis set: a strategy employed by V1? Vision Res, 1997, 37: 3311-3325

20 Olshausen B A, Field D J. Natural image statistics and efficient coding. Netw-Comput Neural Syst, 1996, 7: 333-339

21 Li M Q. Optimization theory and algorithms for image denoising and representation layer by layer. Dissertation for Ph.D. Degree. Beijing: University of Chinese Academy of Sciences, 2017 [李明强. 图像去噪与逐层表达的优化理论 与算法研究. 博士学位论文. 北京: 中国科学院大学, 2017]

22 Conte D, Foggia P, Sansone C, et al. Thirty years of graph matching in pattern recognition. Int J Pattern Recogn Artif Intel, 2004, 18: 265-298

23 Bourgeois F, Lassalle J C. An extension of the munkres algorithm for the assignment problem to rectangular matrices. Commun ACM, 1971, 14: 802-804

24 Birgin E G, Mario M J. Large-scale active-set box-constrained optimization method with spectral projected gradients. Comput Opt Appl, 2002, 23: 101-125

25 Beck A, Teboulle M. Fast gradient-based algorithms for constrained total variation image denoising and deblurring problems. IEEE Trans Image Process, 2009, 18: 2419-2434 


\title{
Fusion of front-end and back-end learning based on layer-by-layer data re-representation
}

\author{
Tiande $\mathrm{GUO}^{1,2}$, Congying $\mathrm{HAN}^{1,2^{*}}$ \& Mingqiang $\mathrm{LI}^{1,3}$ \\ 1. University of Chinese Academy of Sciences, Beijing 100049, China; \\ 2. Key Laboratory of Big Data Mining and Knowledge Management, Chinese Academy of Sciences, Beijing 100190, \\ China; \\ 3. Information Science Research Institute, China Electronics Technology Group Corporation, Beijing 100086, \\ China \\ * Corresponding author. E-mail: hancy@ucas.ac.cn
}

\begin{abstract}
Based on two research contents of machine learning, a two-element layered model of machine learning is proposed. In addition, the concepts of front-end learning, back-end learning, a combination of front-end and back-end learning, and the fusion of front-end and back-end leaning are presented. Specifically, a framework and optimization model for the fusion of front-end and back-end learning is constructed. For front-end learning, which is a simulated hierarchical working mechanism of the brain, we present a layer-by-layer data re-representation method, which is driven by both data and a model. In addition, we propose a specific implementation of the data re-representation method for visual learning.
\end{abstract}

Keywords machine learning, pattern recognition, data representation, hybrid driven by data and model

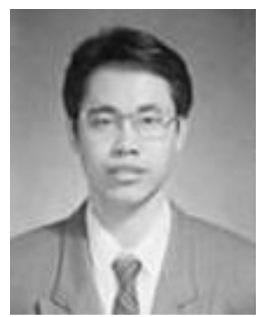

Tiande GUO was born in 1964. He received his M.S. and Ph.D. degrees from the Institute of Applied Mathematics, Chinese Academy of Sciences, Beijing, in 1992 and 1998, respectively. He is currently a professor in the School of Mathematical Sciences, University of Chinese Academy of Sciences, Beijing. His research interests include machine learning, wavelet theory and its applications, pattern recognition, wireless sensor networks, and operations research.

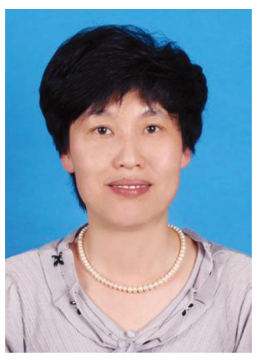

Congying HAN was born in 1972. She received a $\mathrm{Ph} . \mathrm{D}$. degree from the School of Mathematical Sciences at Shanghai Jiao Tong University, Shanghai, in 2008. She is currently a professor at the School of Mathematical Sciences, University of Chinese Academy of Sciences, Beijing. Her research interests include machine learning, optimization theory and methods, and pattern recognition.

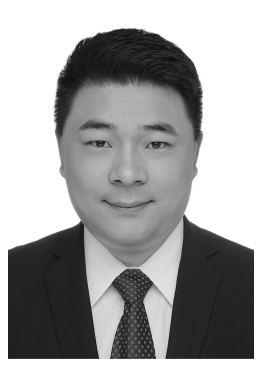

Mingqiang LI was born in 1988. He received a $\mathrm{Ph}$.D. degree from the School of Mathematical Sciences at the University of Chinese Academy of Sciences, Beijing, in 2017. Currently, he is a researcher at the China Electronics Technology Group Corporation. His research interests include machine learning, optimization theory and methods, and pattern recognition. 